\title{
SARAH, outil de traitement des images satellite et radar
}

\section{SARAH, a method of processing satellite and radar pictures}

\author{
J. Tardieu \\ Direction de la Météorologie \\ Etablissement d'Etudes et de Recherches Météorologiques \\ Centre de Recherche en Physique de l'Atmosphère \\ 78470 Magny-les-Hameaux
}

Les radars et les satellites sont d'excellentes plateformes pour observer à distance et suivre les principaux processus météorologiques qui se développent sous nos latitudes. Traditionnellement, ces observations sont analysées séparément et corrélées mentalement pour la prévision et la recherche en intégrant en outre les données du réseau météorologique.

$\mathrm{La}$ restitution sur un même maillage, des données provenant des satellites et des radars avait été proposée dans le cadre du projet HYPLEX par Reynolds et Smith (1979) qui ont montré l'intérêt pour la recherche et la prévision du traitement interactif des images. A l'EERM, dans le cadre du traitement des données acquises pendant l'expérience LANDES 1979, nous avons développé une chaîne permettant de restituer dans un même quadrillage géographique des champs issus de capteurs à grand coup d'observation (radar à acquisition numérique ou satéllite...) ou de réseaux de capteurs (réseaux de pluviomètres... ).

\section{Description de la chaîne}

Pour réaliser cette chaîne, nous avons cherché à utiliser de manière judicieuse, les importantes possibilités te chniques qui existent à l'EERM, entre autre l'existence d'un calculateur puissant, le Control Data CYBER 1975, le Centre de calcul de l'Observatoire de Magny avec les divers systèmes de visualisation et surtout le système interactif de traitement des images METEOSCOPE. La chaîne doit être d'un emploi simple et facilement évolutive en fonction des disponibilités ou de l'évolution des matériels.

Le graphique de la figure 1 montre les principales étapes du traitement. La partie principale est un fichier numérique, bi ou tridimensionnel, en point de grille d'un format fixe :

- en entrée: les données préalablement enregistrées sur bande magnétique numérique,

- Ière étape : la restitution des données sur une grille

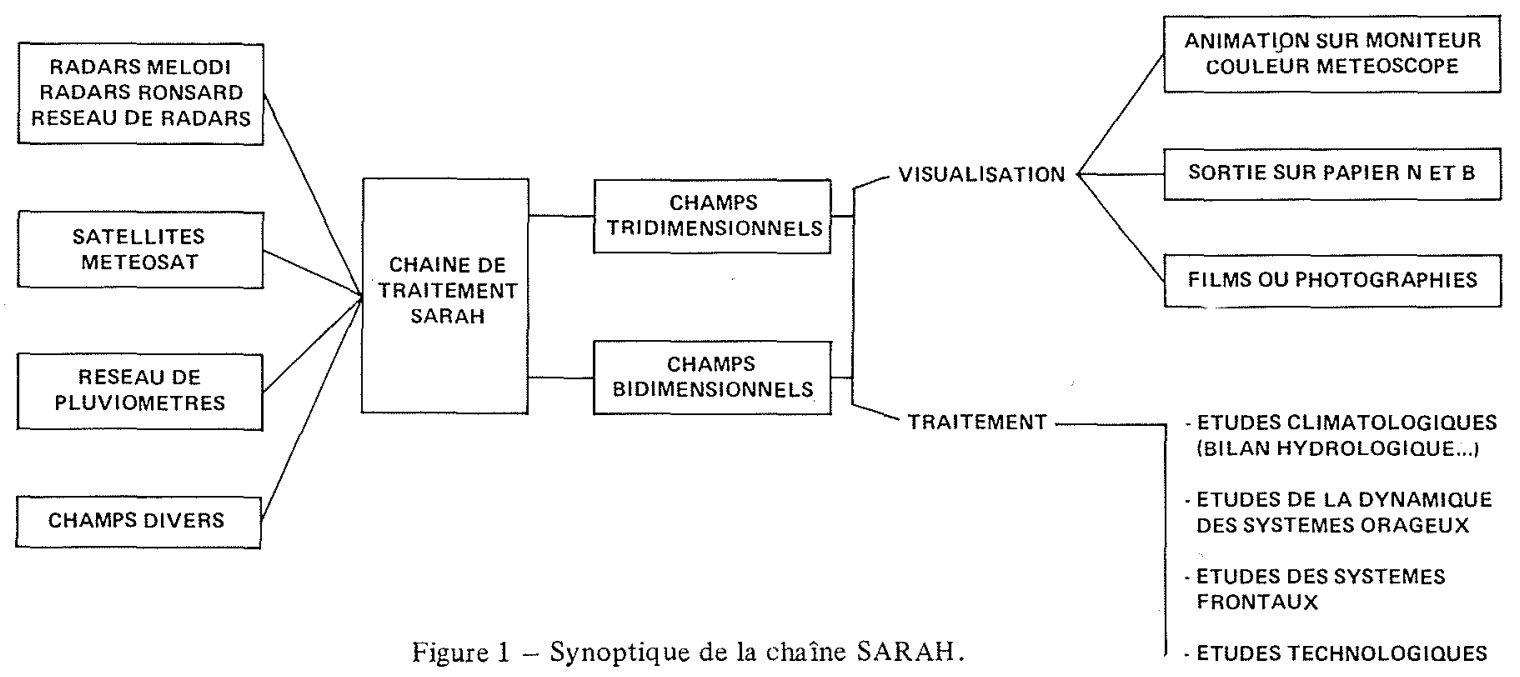

LA HOUILLE BLANCHE/N ${ }^{\circ}$ 5/6-1983 
décrite suivant une projection géographique classique,

$-2^{e}$ étape :

- interprétation des résultats

- visualisation de ces champs

Reprenons en détail ces étapes:

Entrée : actuellement sont réalisés

- traitement des données du satellite géostationnaire METEOSAT

- radar à acquisition numérique

MELODI du réseau météorologique Français

RONSARD du CRPE/CNET

- en cours de développement, réseau de pluviomètres.

\section{La grille}

La grille utilisée est pour l'instant décrite suivant les projections Lambert ou Mercator qui sont des projections universellement utilisées avec la projection stéréographique Polaire: la Projection Lambert est notamment utilisée par l'IGN pour ses cartes, car elle minimise les déformations sous nos latitudes. La projection Mercator sert essentiellement dans le cadre de la campagne africaine COPT 81.

Actuellement nous améliorons les programmes pour utiliser la projection stéréographique polaire, projection couramment employée par les météorologistes européens.

\section{Les programmes}

Les modules METEOSAT ont été développés par Claude Pastre et Jean-Paul Jullien, en utilisant un algo-

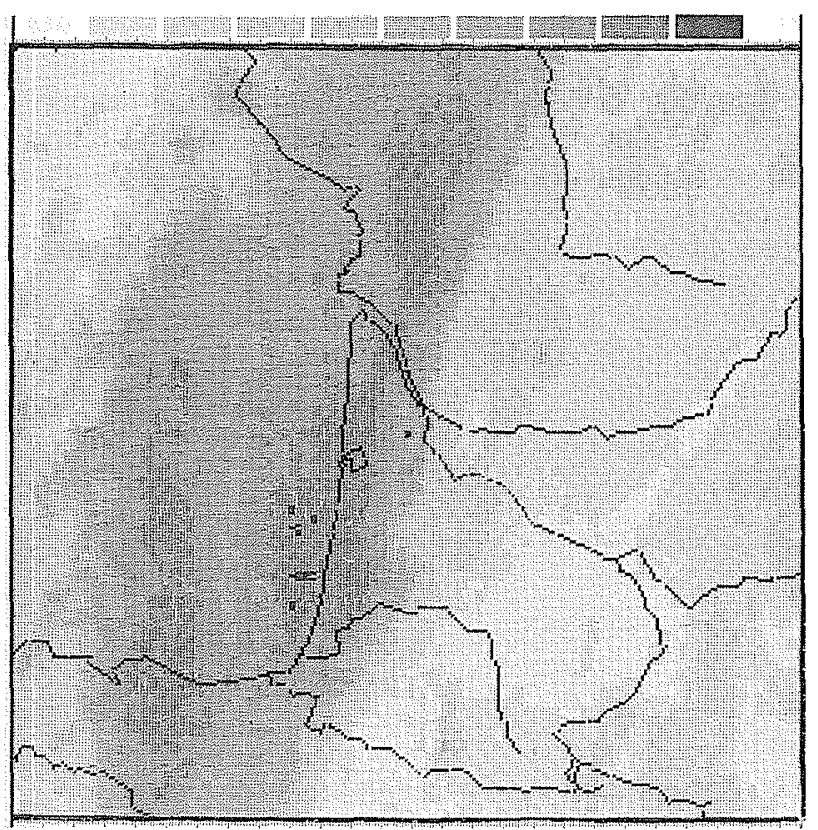

Figure 2 - METEOSAT canal thermique à 1130 TU. L'image est restituée sur une projection Lambert $30 / 60$, centrée sur Bordeaux-Mérignac. Le pixel mesure $2 \mathrm{~km}$ de côté au centre de l'image. Les teintes de gris correspondent à la température de rayonnement au niveau du satellite. rithme qui consiste à découper le champ satellitaire en plusieurs sous-secteurs facilement traitables par le CYBER 175.

Pour les données radar, la chaîne ne traitant que les grandeurs scalaires, seules les réflectivités sont prises en compte. L'algorithme tient compte de la forme de la terre, des conditions standards de propagation des ondes radioélectriques et interpole angulairement les données lorsque la vitesse de rotation de l'antenne est trop élevée.

Les modules construisent un champ de 256 lignes de 256 points, bi ou tri dimensionnel sur 12 niveaux suivant le type d'exploitation effectué par le radar et le but recherché.

Enfin, sont paramétrables : les dimensions de la maille (suivant les trois axes), la position du centre de la grille, l'altitude de la base de la grille ... Le fichier résultat possède une structure simple, pouvant contenir plusieurs champs acquis simultanément ou pendant l'intervalle de temps considéré. Un certain nombre de routines permet d'accéder aisément à des données, notamment en effectuant des coupes.

Diverses possibilités de visualisations ont été développées notamment sur imprimantes électrostatiques. Ces techniques simples d'emploi, permettent un contrôle rapide des traitements. Mais le traitement le plus souple consiste à utiliser le METEOSCOPE, système de traitement interactif des images sous forme numérique, développé et commercialisé par la Société NUMELEC/SEIN, sous le nom de PERICOLOR 1000.

Dans l'état actuel, le système comprend :

- un moniteur de télévision de $51 \mathrm{~cm}$

- 8 plans mémoire de $256 * 256$ pixels de 8 bits (ou 4 de 16 bits)

- 4 plans marqueurs de 1 bit



Tigure 3 -- Echos enregistrés par le radar MELODI de BordcauxMérignac à 1151 TU. L'image est restituée sur la même projection que pour la figure 2. La chaîne numérique SAPHYR, dans la configuration utilisée, acquiert les informations entre 10 et 138 Kilomètres (limites matérialisées par des cercles). Les échos à l'est du radar sont des échos non météorologiques (ćchos fixes). 


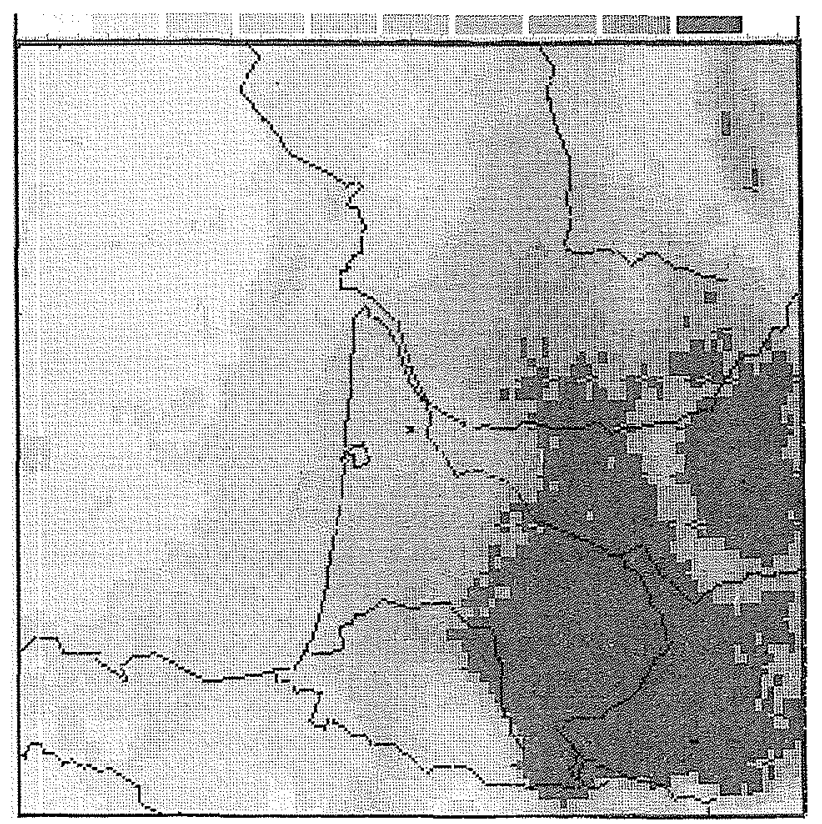

Figure 4-METEOSAT canal thermique à $1630 \mathrm{TU}$ (voir Fig. 2).

- 1 plan alphanumérique de 36 lignes de 50 caractères permettant l'affichage des commentaires et le dialogue avec le système.

- 1 microprocesseur INTEL 8080A, disposant d'une mémoire morte de 32 Koctets et d'une mémoire vive de 8 Koctets. Il permet, grâce à un logiciel d'emploi simple, de gérer les plans images, marqueurs et alphanumériques, ainsi que le traitement des images (zoom, décadrage, vrai/fausse couleur, conversion contenu/ couleurs, animation, opérations arithmétiques et booléennes entre deux ou plusieurs images ou sousimages....).

- 1 liaison avec un ordinateur, actuellement un HP 2100, qui possède entre autre deux disques de 5 Moctets, 4 dérouleurs de bandes magnétiques, une mémoire de 64 Koctets... Cette liaison permet le transfert d'images dans les deux sens, l'écriture des plans marqueurs et caractères ainsi que la modification des paramètres pour la gestion des images.

Cette chaîne a permis l'analyse des situations météorologiques au cours de la campagne des LANDES 1979 : le 15 août. Rappelons (voir le compte-rendu de la campagne LANDES 79) que, ce jour-là, un front froid est passé sur la France, provoquant des orages (Figs 2 et 4). La figure 2 montre la région des Landes, en coordonnées Lambert, le pixel mesure $2 \mathrm{~km}$ au centre de l'image

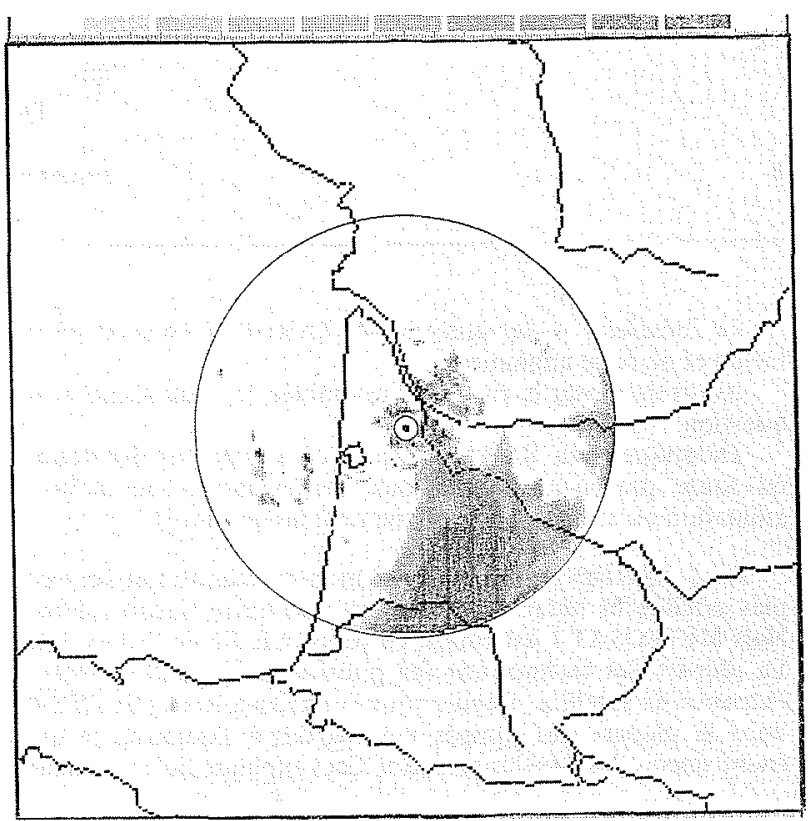

Figure 5 - Echos enregistrés par le radar MELODI à 1642 TU (voir figure 3 ).

qui a $500 \mathrm{~km}$ environ dans les deux directions. Nous pouvons distinguer les nuages froids (ici en noir) qui abordent la zone d'expérience. La figure 3 montre, dans le même repère, les précipitations visualisées par le radar MELODI de Bordeaux, précipitations en bande. Ceci permet, entre autre, de contrôler la validité des diverses méthodes proposées pour estimer la pluie à partir des données satellitaires, mais surtout d'analyser finement la structure des bandes de pluie et leur évolution.

Les figures 4 et 5 ont été prises vers $1650 \mathrm{TU}$. La région est recouverte par une zone orageuse de plus de $200 \mathrm{~km}$. Sur la photo METEOSAT, canal thermique, la zone orageuse est matérialisée par les nuages froids. Sur l'analyse radar, nous pouvons remarquer que les précipitations sont discontinues; elle proviennent de cellules discrètes qui évoluent rapidement dans le temps.

\section{Références}

Compte-rendu de l'expérience LANDES 79. - Documents COPT $\mathrm{N}^{\circ} 3155 \mathrm{pp}$.

REYNOLDS D. et SMITH E. - Detailled Analysis of composited digital data. Bull. of $A M S$ vol 60 pp. 1024-1037, 1979.

LOVEJOY S., TARDIEU J. et MONCEAU G. - Etude d'une situation frontale. Analyse météorologique et fractale. La Météorologie num. spéciale Juillet 1982. 


\section{Discussion}

Président $:$ R. HLAVEK

Le Président. - Je remercie M. TARDIEU de cette présentation et de cette animation.

Avant de passer la parole à l'assemblée, je voudrais poser une question.

Théoriquement, SARAH permet de superposer des données de toute provenance. Avez-vous des exemples, au moins en animation ou en projection, d'une telle intégration?

M. J. TARDIEU. - Je vous ai montré aujourd'hui des exemples satellite et radar. C'est par cela que notre travail a débuté. Mais METEOSAT 1 est tombé en panne à la fin de l'année 1979. La plupart des travaux ont été poursuivis en 1980 et 1981 en l'absence de satellite. Depuis vous connaissez le sort de l'EERM, dont la plupart des équipes sont parties à Toulouse, ce qui a freiné encore les développements. Ceci explique qu'actuellement on ne puisse faire autre chose de plus que l'analyse des données issues de modèles numériques de prévision type PERIDOT. Cette méthode permet d'analyser le comportement des modèles conjointement avec les données satellite et radar.

D'autre part, la plupart des idées qui ont été montrées ici sont reprises dans le cadre de METEOTEL. La palette de couleurs, la facon de présenter les données, la superposition satellite et radar sont maintenant utilisées de manière opérationnelle à la date d'aujourd'hui.

L'objet de SARAH est de reprendre après-coup une partie des données pour les traiter de manière plus complète dans une chaîne d'étude. Ainsi, les précipitations du 14 octobre 1980 ont fait l'objet d'une étude plus complète, notamment pour mettre en évidence la variation temporelle des précipitations dont $M$. LOVEJOY a beaucoup parlé.

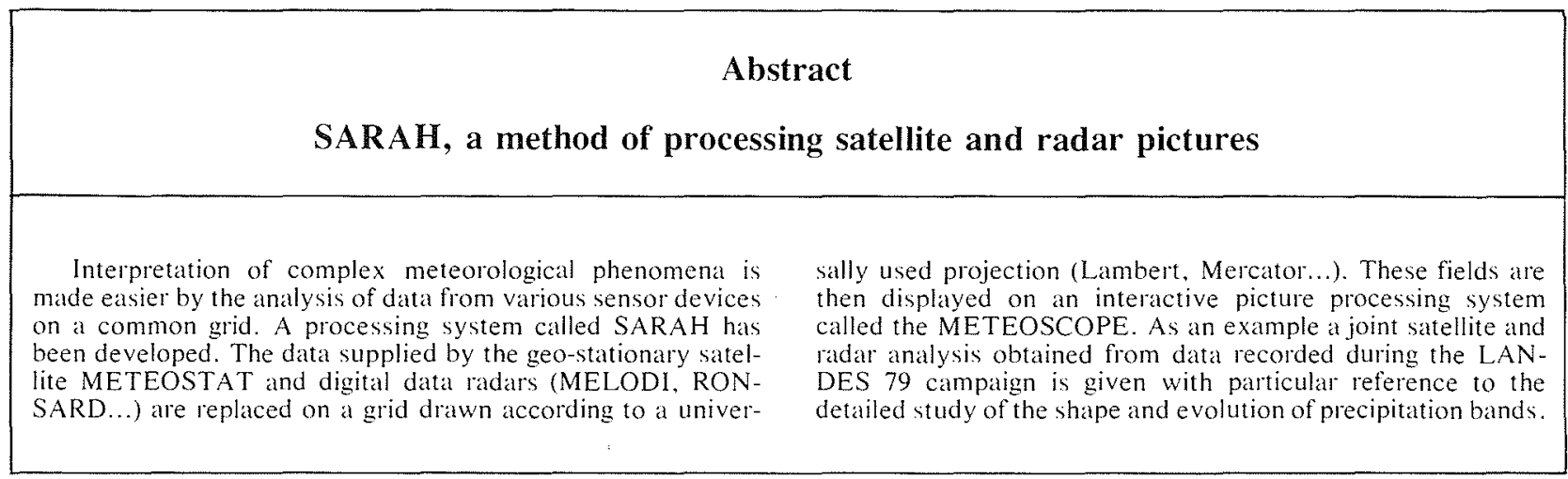

